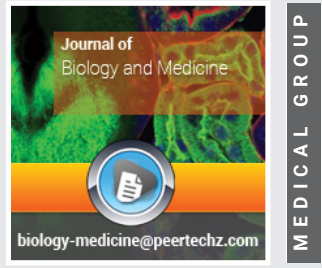

\title{
Correlation of glycosylated hemoglobin level with the severity of coronary artery disease in diabetic patients
}

\author{
Hossam Eldin M Mahmoud ${ }^{1}$, Abdel Rahman A Elsaied ${ }^{2}$ and \\ Mohammed H Hassan ${ }^{3 *}$
}

'Internal Medicine Department, Faculty of Medicine, South Valley University, Qena, Egypt

${ }^{2}$ Clinical Pathology Department, Faculty of Medicine, South Valley University, Qena, Egypt

${ }^{3}$ Medical Biochemistry Department, Faculty of Medicine, South Valley University, Qena, Egypt

Received: 18 December, 2019

Accepted: 03 February, 2020

Published: 05 February, 2020

*Corresponding author: Dr. Mohammed H Hassan, Associate Professor of Medical Biochemistry, Faculty of Medicine, South Valley University, Qena, Egypt, Tel: +201098473605;

E-mail: Mohammedhosnyhassaan@yahoo.com; mohammedhosnyhassaan@med.svu.edu.eg

Keywords: HbA1c; Coronary artery disease; Diabetes mellitus

https://www.peertechz.com

\section{Check for updates}

\section{Abstract}

Background: Diabetes Mellitus (DM) is known to cause microvascular and possibly macrovascular complications as it one of the major risk factors for Coronary Artery Disease (CAD). CAD is a major cause of death in developed countries. The study aims to assess the relation of glycated Hemoglobin (HbA1c) levels and severity of $C A D$ in diabetic patients.

Patients and methods: The study included 200 patients with type II diabetes mellitus and symptoms suggesting CAD in whom coronary angiography was indicated in internal medicine department, Qena University hospitals. Full medical history was taken with special concern on presence of associated co-morbidities (Hypertension, dyslipidemias, smoking history). They were assessed according to HBA1C level and Gensini score. Electroencephalogram and transthoracic echocardiography were performed to the included patients. Patients were divided into 6 groups according to HBA1C starting from 4 to $>14$ and 7 groups according to Gensini score from 0 to 140 , 3 groups according EF\% (Ejection fraction) from $<40 \%$ to $>50 \%$.

Results: Our study included 132 females with mean age $52.33 \pm 9.618$ and 68 males with mean age $55.04 \pm 10.147$, One hundred and eleven patients were hypertensive, 68 patients were smoker, dyslipidemia found in 116 patients, 84 patients had ECG ischemic changes and RSWMA (Segmental wall motion abnormality at rest) by transthoracic echocardiography found in 88 patients. A statistically significant positive correlations between HBA1C with Gensini score, smoking, dyslipidemia, hypertension were present $(p<0.05)$.

Conclusion: HbA1c can be used as a predictor of severity of coronary artery disease measured by Gensini score as patients with a higher level of HbA1c had significant severe lesions with higher Gensini scores.

\section{Introduction}

Ischemic Heart Disease (IHD) is a major cause of death in developed countries, IHD remains responsible for about one-third of all deaths in individuals over age 35, Diabetes Mellitus (DM) is known to cause microvascular and possibly macrovascular complications as it one of the major risk factors for Coronary Artery Disease (CAD) [1-3].

Most of the mortality in patients with DM is due to atherosclerotic disease. Also, in the risk for cerebrovascular disease, peripheral vascular disease, and heart failure are associated with diabetes. Observations from many clinical trials showed that DM is associated with a higher prevalence of CAD with higher morbidity and more fatal coronary events due to a higher incidence of plaque rupture and superimposed thrombosis in diffusely diseased coronary arteries [4].

The American Diabetes Association stated that lowering HbA1c is associated with a less neuropathic and microvascular complications, and may be also associated with less macrovascular complications of DM. However, they suggested 
that more studies should be done to establish the relationship between HbA1c and macrovascular complications of DM [5].

\section{Aim}

To study the possible correlations of HbA1c with the severity of CAD among patients with T2DM using Gensini score.

\section{Patients and methods}

\section{Patients}

This study included 200 patients (132 females and 68 males) in Qena University Hospitals from December 2018 till September 2019, who were previously diagnosed to have diabetes mellitus according to American Diabetes Association Diagnostic Criteria for Diabetes Mellitus 2018 and ischemic heart disease either by history of recurrent attacks of typical chest pain, previous unstable angina or myocardial infarction, ECG different criteria of ischemia or Echo showing regional wall motion abnormalities. We excluded patients with alcohol intake, lead, opiate toxicity, splenectomy and uremia as they had an increased level of HbA1c.

\section{Data collections}

\section{All of the patients had been subjected to the following:}

- Full history with stress on risk factors of coronary artery disease, exclusion criteria, and detailed physical examination.

- 12 lead resting ECG, HbA1c measurement, Lipid profile, 2D transthoracic Echocardiography (TTE), Diagnostic coronary angiography all images were recorded digitally [6].

- Assessment of the severity of coronary artery disease had been done by using Gensini score:

o Gensini score grades narrowing of the lumen of the coronary artery and scores it with numerical values with the following method: $<25 \%$ narrowing (1 point), $26-$ $50 \%$ ( 2 points), $51-75 \%$ ( 4 points), $76-90 \%$ (8 points), $91 \%-99 \%$ (16 points), and 32 points for $100 \%$ occluded artery.

o This score is then multiplied by a factor according to the importance of the coronary artery as follows: 5 for a Left Main Stem (LMS) lesion, 2.5 for proximal Left Anterior Descending artery (LAD) and proximal Circumflex artery (CX) lesions, 1.5 for a mid-LAD lesion, 1 for distal LAD, mid/distal CX and right coronary artery lesions and 0.5 for any other branch.

o The individual scores of all lesions then summated to give the total Gensini score [7].

\section{Laboratory workup}

After 10-12hours fasting, 6 cc venous blood was withdrawn from every included subject and divided onto two parts; $3 \mathrm{ml}$ placed on EDTA-containing tube for immediate HbA1c assay using cobas c311, Hitachi, Roche Diagnostics, Germany.
The other $3 \mathrm{ml}$ was evacuated into a plain vacutainer tube where blood was allowed to clot for 10-20minutes at room temperature before centrifugation at a speed of $3000 \times \mathrm{g}$ for 5minutes, then serum was used for lipid profile assay: HDL, triglycerides and total cholesterol were measured according to the manufacture protocol using a spectrophotometric assay kit provided by BIOLABO SAS, Les Hautes Rives 02160, Maizy, France. The serum level of LDL cholesterol was calculated.

\section{Statistical analysis}

All patients had been analyzed using the Statistical Package for Social Sciences (SPSS) version 25(IBM corporation, Chicago, USA, 2017). Quantitative data was expressed as mean and standard deviation, while qualitative data was expressed as number and percentages. Pearson Correlation coefficient was used to estimate the correlation between two quantitative variables and multivariate correlation was used to estimate the possible role of Gensini score in the estimation of high HbA1c, ECG changes and lower EF. $P$ value was considered significant if $<0.05$ and highly significant if $<0.001$.

\section{Ethics}

Written consents from patients who participated in the study or from their families were obtained and approved by Qena Medical Ethics Committee (QMEC) of Faculty of Medicine- South Valley University. The study was conducted in accordance with the Declaration of Helsinki.

\section{Results}

Our study included 200 patients, 132 females with mean age $52.33 \pm 9.618$ and 68 males with mean age $55.04 \pm 10.147$ (Figure 1).

In our study we found that 111 patients were hypertensive, 68 patients were smokers, dyslipidemia found in 116 patients, 84 patients had ECG ischemic changes and RSWMA (Segmental wall motion abnormality at rest) by transthoracic echocardiography found in 88 patients.



Figure 1: Age groups involved in the study. 
Patients were divided into 6 groups according to HBA1C starting from 4 to $>14$ and 7 groups according to Gensini score from 0 to 140,3 groups according EF\% (Ejection fraction) the $1^{\text {st }}<40 \%$ (28 patients), $2^{\text {nd }} 40: 50 \%$ ( 55 patients) and the $3^{\text {rd }}>50 \%$ (117 patients).

HbA1c was variably distributed among patients. HbA1c between $(4-6)$ was found in 31 patients, between $(6-8)$ in 23 , between $(8-10)$ in 17 , between $(10-12)$ in 38 , between $(12-14)$ in 56 , and $>14$ in 35 patients (Figure 2 ).

Gensini score was also variably distributed among patients, 39 patients had Gensini score (0-20), 32 had score (21-40), 23 had score (41-60), 15 had score (61-80), 31 had a score between (81- 100) while 38 patients have Gensini score of (101- 120) and 22 patients had score of (121-140) (Figure 3).

There was a statistically significant positive correlation between Gensini score and Age (as older patients had higher Gensini score $P$-value 0.03), smoking (as smoker patients had higher Gensini score, $P$-value <0.01), Dyslipidemia (as dyslipidemic patients had higher Gensini score, $P$-value $<0.01$ ), hypertension (as hypertensive patients had higher Gensini score, $P$-value $<0.01$ ), Ejection fraction (as patients with lower EF\% had higher Gensini score, $P$-value $<0.01)$, RSWMA by TTE and Ischemic changes in their ECG had higher Gensini score, $P$-value $<0.01$

There was a statistically significant positive correlation between HBA1C and Gensini score as the HbA1c increased the Gensini score increased ( $P$-value $<0.01)$. (Figure 4 ).

In our study we found that there was multivariate significant correlation according to Gensini score between HBA1C level, ECG finding and Ejection fraction (EF\%) by transthoracic Echocardiography as patients with higher Gensini score found to have higher HBA1c level, ischemic changes in their ECG and lower ejection fraction $(P$-value <0.01) (Figure 5, Table 1).

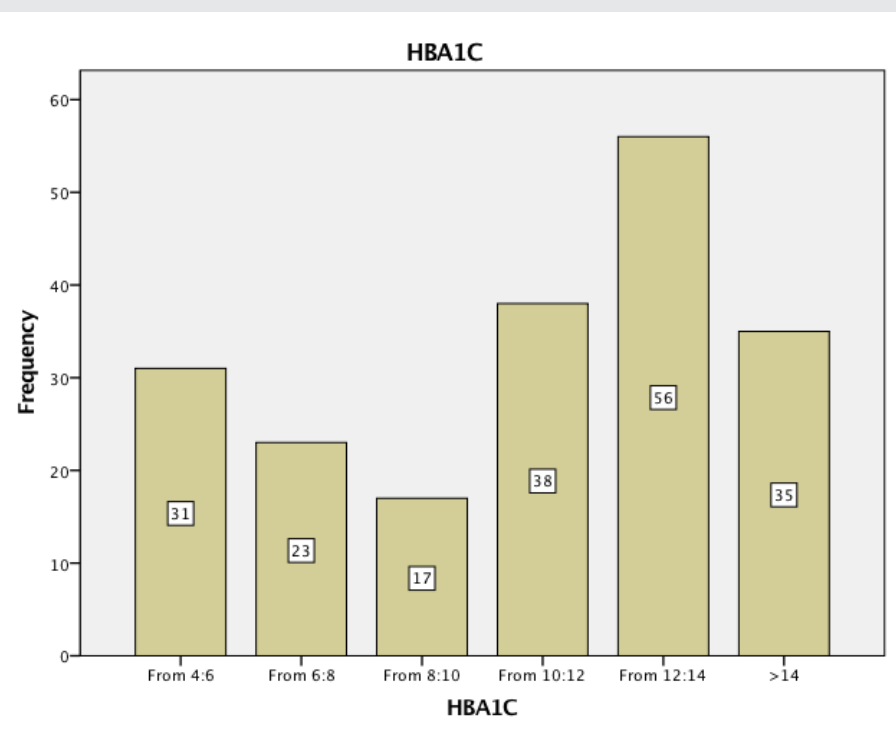

Figure 2: HBA1C groups involved in the study.

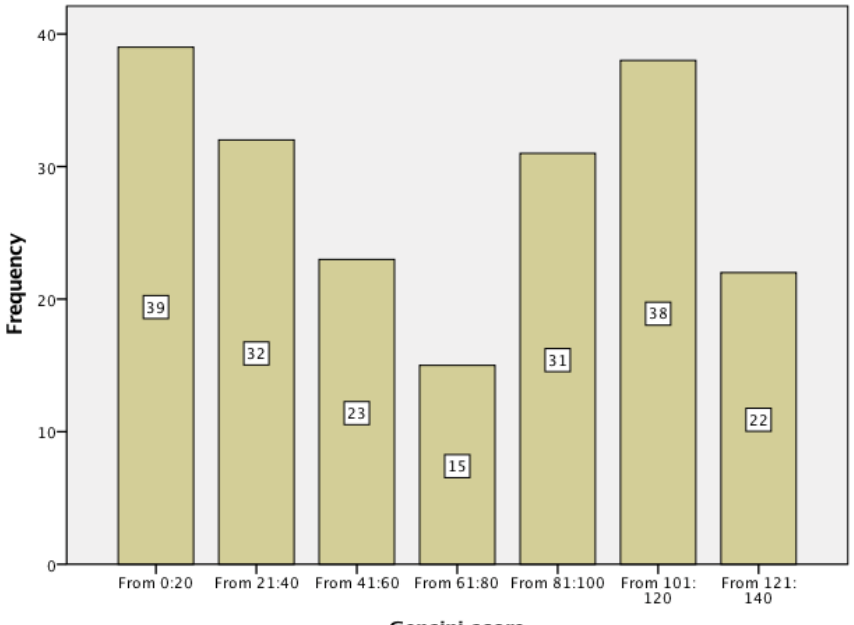

Gensini score

Figure 3: Gensini score groups involved in the study.

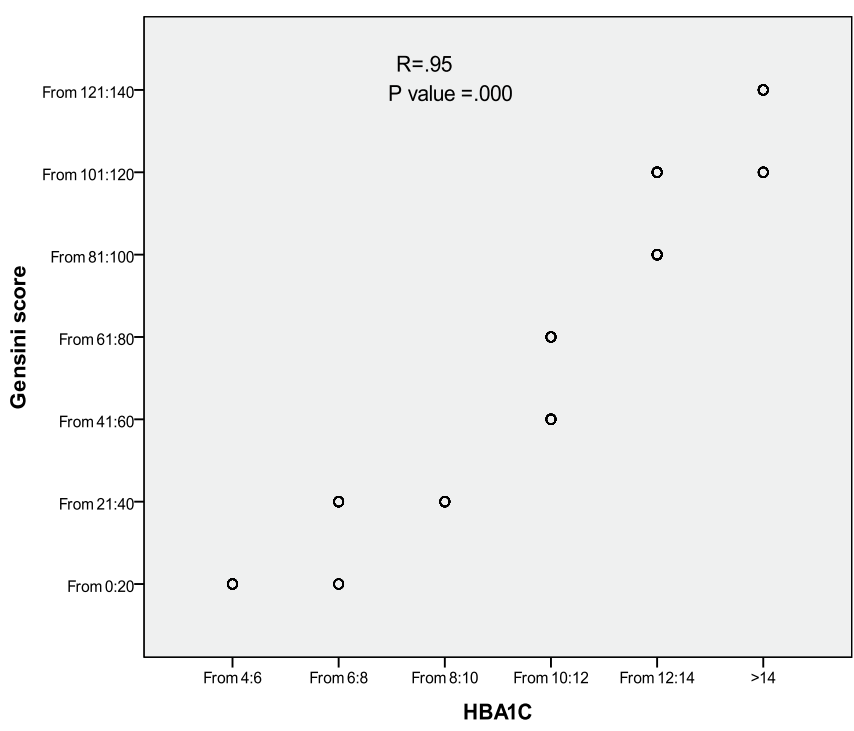

Figure 4: Correlation between HBA1C and Gensini score.

\section{Discussion}

Cardiovascular Diseases, including CAD, are major causes of morbidity and cardiovascular mortality in patients with type 2 diabetes mellitus (T2DM) [3].

Diabetic patients usually have many health issues that contribute to the higher risk of cardiovascular diseases including hyperglycemia, fluctuation of blood glucose, central obesity, hyperlipidemia and hypertension [1].

In this present study, we determine the relationship of HbA1c with the severity of coronary artery disease.

Our results were in agreement with many researchers [8-13], who reported a positive correlation between severity of CAD and HbA1c. A study by Saleem, et al., [9], they found that there was statistically significant positive correlation according to Gensini score and DM $(p=0.003)$, hypertension 


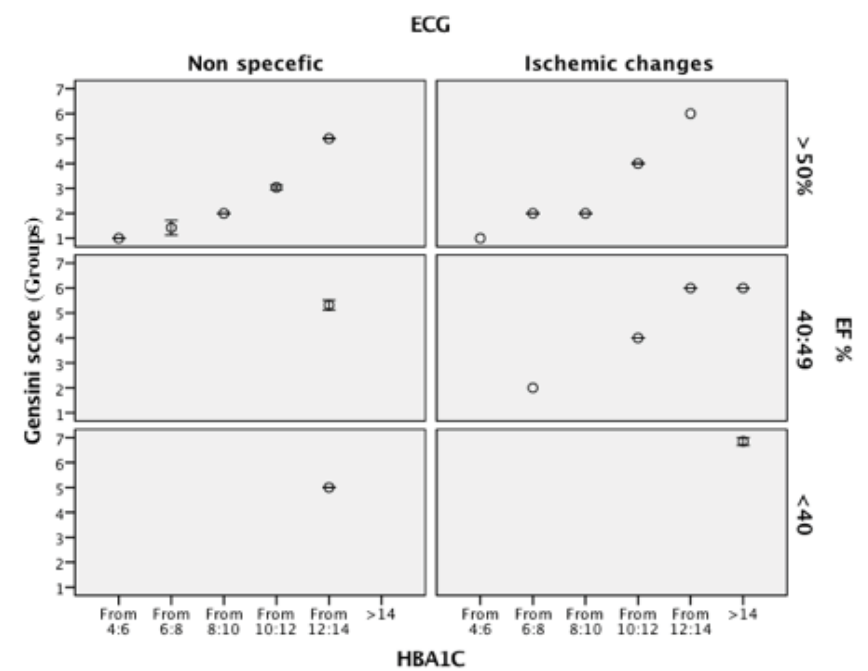

Figure 5: Multivariate correlation according to Gensini score between HBA1C level, ECG finding and (EF\%).

Table 1: Multivariate correlation according to Gensini score between HBA1C level, ECG finding and (EF\%)

\begin{tabular}{|c|c|c|c|}
\hline \multicolumn{4}{|c|}{ Parameter Estimates } \\
\hline & Estimate & Std. Error & Sig. \\
\hline HBA1C & 9.431 & 1.202 & $<0.0001$ \\
\hline EF\% & 2.034 & .576 & $<0.0001$ \\
\hline ECG & 4.004 & .737 & $<0.0001$ \\
\hline
\end{tabular}

$(p=0.018)$, there was a positive significant correlation between Gensini score and HbA1c ( $r=0.427, p<0.001)$, and that HbA1c was an independent factor influencing the severity of coronary artery disease that result match the result of our present study.

$\mathrm{Su}$, et al., [10], studied the association of glycemic variability and the presence and severity of CAD in 252 patients with type 2 diabetes and reported that Gensini score closely correlated HbA1c level $(p=0.022)$ also, Gensini score was significantly increased with age as we found in our present study. Ravipati, et al., [8], studied the association of HbA1c level with the severity of $\mathrm{CAD}$ in 315 diabetic patients who underwent coronary angiography because of chest pain and stated that HbA1c level significantly increased with the increasing severity of the diseased arteries that meet our results.

A study by Jia, et al., [11], reported significant differences between $\mathrm{CAD}$ and non-CAD subjects with respect to age $(\mathrm{p}=0.002)$, sex $(\mathrm{p}=0.001)$, total cholesterol $(\mathrm{p}=0.042)$, fasting glucose $(p=0.005), \mathrm{HbA1c}(p=0.000)$ and HbA1c score $(p=0.000)$, However, there was no statistically difference in triglyceride $(p=0.319), \quad \operatorname{HDL}-c(p=0.714)$ and LDL-c $(p=0.905)$ levels, smoking status $(p=0.079), \operatorname{SBP}(p=0.155)$ and DBP $(p=0.303)$ between $\mathrm{CAD}$ and non-CAD subjects.

In our present study we found that there was statistical significance positive correlation according to Gensini score with respect to lipid profile ( $P$ value $<0.01)$, hypertension $(P$ value $<0.01)$ and smoking $(P$ value $<0.01)$. In agreement with our findings, a study has been done by Gaber, et al., [12], there were 100 diabetic patients were scheduled for coronary angiography ,the analysis of the result showed that there was a statistically significant positive correlation between HbA1c levels \& Gensini scores, with increasing HbA1c values there was an increase in Gensini score $p$-value $<0.001$. There was a statistically significant positive correlation between age \& Gensini scores, with increasing age there was an increase in Gensini score p-value $<0.016$, all these results match the result of our study. But in the same study, they found that there was no statistically significant difference between hypertensives and non-hypertensives, dyslipidemic and non-dyslipidemic and smokers and non-smokers regarding their HBA1c levels ( $p$-value $>0.05$ ) on the contrary of the result of our study which found a positive correlation of all these factors and Gensini score.

In line with our study, the study of Hong, et al., [13], they found that $\mathrm{HbA1C}$ was an independent predictor of severity of CAD $(P<0.001)$ even after adjusting for age, gender, traditional risk factor of $\mathrm{CAD}$, lipid profile and fasting blood glucose.

On the contrary to our results and the previous studies Schnyder, et al., [14], studied the association of HbA1c with the severity of CAD in 631 patients (148 diabetic patients and 483 non-diabetic patients) and he discovered that the severity of CAD was not associated with HbA1c level. This discordance may be attributed to the inclusion of non-diabetic patients representing the major cohort of the study in contrast to our patients who all were diabetics; also the difference of the sample size between our study and this study may be a contributing factor.

\section{Conclusion}

$\mathrm{CAD}$ is a major cause of disability and premature death throughout the world. HbA1c can be used as a predictor of severity of coronary artery disease measured by Gensini score as patients with a higher level of HbA1c had significant severe lesions with higher Gensini score. Further studies on a larger number of patients and on other patients' categories are recommended.

\section{Study limitations}

In addition to the relatively small number of patients and being a single-center study so that some subgroup comparisons may have lacked the power to detect significant differences for selected variables, the major limitation of our study is that it did not include many patients' categories like those addict to alcohol, having uremia, lead and opiate toxicity or those who surgically removed their spleen as they have false increased levels of HbA1c, another limitation due to the fact that this study was a cross-sectional one, so our results only could show the association between HBA1c and prevalent CAD but not incident CAD. Our study didn't use a glycemic variation (fasting blood sugar, postprandial glucose excursion, mean amplitude of glycemic excursion MAGE) as a tool of prediction of severity of CAD. Finally, our study lacked the value of HbA1c for prognostic outcomes in patients with AMI or ACS after CABG or stent implantation as we were limited by the short time to have a significant result. 


\section{References}

1. Rosamond W, Flegal K, Furie K, Go A, Greenlund K, et al. (2008) Heart disease and stroke statistics--2008 update: a report from the American Heart Association Statistics Committee and Stroke Statistics Subcommittee. Circulation 117: e25-146. Link: http://bit.ly/39cykjB

2. Lloyd-Jones D, Adams RJ, Brown TM, Carnethon M, Dai S, et al. (2010) Executive summary: heart disease and stroke statistics--2010 update: a report from the American Heart Association. Circulation 121: 948-954. Link: http://bit.ly/2Ur2U4T

3. Nichols M, Townsend N, Scarborough P, Rayner M (2014) Cardiovascula disease in Europe 2014: epidemiological update. Eur Heart J 35: 2950-2959. Link: http://bit.ly/3baCyKc

4. Donnelly R, Emslie-Smith AM, Gardner ID, Morris AD (2000) ABC of arterial and venous disease: vascular complications of diabetes. BMJ 320: 1062-1066. Link: http://bit.ly/2UumxZF

5. American Diabetes Association (2007) Nutrition Recommendations and Interventions for Diabetes: a position statement of the American Diabetes Association. Diabetes care 30: S48-S65. Link: http://bit.ly/2vReZGc

6. Boucher RA, Myler RK, Clark DA, Stertzer SH (1988) Coronary angiography and angioplasty. Catheterization and cardiovascular diagnosis 14: 269-285. Link: https://go.aws/31tPhmF

7. Gensini GG (1983) A more meaningful scoring system for determining the severity of coronary heart disease. Am J Cardiol 51: 606. Link: http://bit.ly/3bfm3wn
8. Ravipati G, Aronow WS, Ahn C, Sujata K, Saulle LN, et al. (2006) Association of hemoglobin $A(1 c)$ level with the severity of coronary artery disease in patients with diabetes mellitus. Am J Cardiol 97: 968-969. Link: http://bit.ly/371eia0

9. Saleem T, Mohammad KH, Abdel-Fattah MM, Abbasi AH (2008) Association of glycosylated haemoglobin level and diabetes mellitus duration with the severity of coronary artery disease. Diab Vasc Dis Res 5: 184-189. Link: http://bit.ly/31poEPQ

10. Su G, Mi S, Tao H, Li Z, Yang H, et al. (2011) Association of glycemic variability and the presence and severity of coronary artery disease in patients with type 2 diabetes. Cardiovasc Diabetol 10: 19. Link: http://bit.ly/31pMbA8

11. Jia EZ, An FH, Chen ZH, Li LH, Mao HW, et al. (2014) Hemoglobin A1c risk score for the prediction of coronary artery disease in subjects with angiographically diagnosed coronary atherosclerosis. Cell Physiol Biochem 34: 672-680. Link: http://bit.ly/381ku3

12. Gaber AH, El-Kholy TMA, Aziz EA, Helmi MM (2014) Glycosylated Haemoglubin Level and Severity of Coronary Artery Disease. Med J Cairo Univ 82: 87-93. Link: http://bit.ly/380voX4

13. Hong LF, Li XL, Guo YL, Luo SH, Zhu CG, et al. (2014) Glycosylated hemoglobin $\mathrm{A} 1 \mathrm{c}$ as a marker predicting the severity of coronary artery disease and early outcome in patients with stable angina. Lipids Health Dis 13: 89. Link: http://bit.ly/36XMXFN

14. Schnyder G, Pin R, Roffı M, Flammer Y, Hess OM (2001) Association of plasma homocysteine with the number of major coronary arteries severely narrowed. Am J Cardiol 88: 1027-1030. Link: http://bit.ly/396FKES

\section{Discover a bigger Impact and Visibility of your article publication with} Peertechz Publications

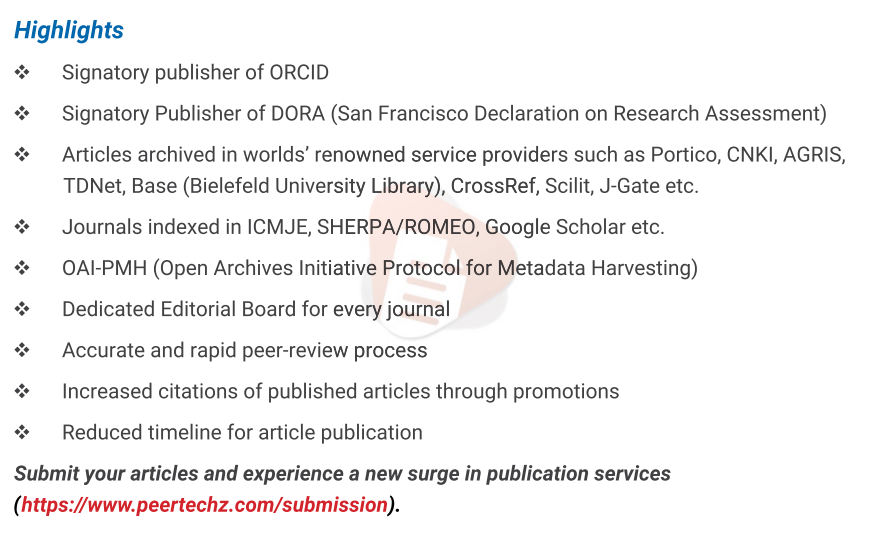

Copyright: @ $2020 \mathrm{M}$ Mahmoud HE, et al. This is an open-access article distributed under the terms of the Creative Commons Attribution License, which permits unrestricted use, distribution, and r eproduction in any medium, provided the original author and source are credited. 